\title{
ESTUDIOS DE POSGRADO EN EDUCACION: EVALUACION DE UNA EXPERIENCIA
}

Gonzalo Cataño*

\section{Introducción}

Los programas de posgrado son un fenómeno reciente en la educación colombiana. Surgieron en la década del 60 y cobraron un desarrollo inusitado durante los años setenta y comienzos de los ochenta. Esta impetuosa aparición era una manifestación directa de la rápida expansión de la educación superior que también había tenido lugar en aquellos años. Si en 1960 la universidad contaba con 23.013 estudiantes, en 1970 tenía 85.560 y en 1980 llegaba a 230.159. Esto es, en veinte años la matrícula universitaria se había multiplicado por diez y sus egresados habían empezado a presionar en la cúspide del sistema educativo por cursos de especialización y por títulos más avanzados.

Esta dinámica obedeció a una constelación de factores técnicos y sociales muy difíciles de separar unos de otros. Las complejidades asociadas a la urbanización y a la industrialización fueron modelando una estructura ocupacional y un sistema de servicios con niveles de especialización apenas conocidos en el medio; proceso al cual se sumó la asimilación del desarrollo técnico y los avances de la ciencia que por aquellos años comenzaron a difundirse por los países del Tercer Mundo. Así, algunas profesiones, como las médicas, se fueron subdividiendo hasta el punto de que hoy no se concibe un médico que no haya alcanzado o esté buscando una especialización. Ello se expresa en los numerosos programas de graduados ofrecidos por las facultades de Medicina del país, que en este momento van desde la Cardiología hasta la Salud Pública, pasando, entre otras, por la Oftalmología, la Dermatología, la Siquiatría, la Radiología y la Pediatría. Lo mismo ocurre con la Ingeniería y con las Ciencias Naturales donde los posgrados son ahora una actividad floreciente. La Ingeniería Química, de Transportes, Sanitaria y de Sistemas, de la misma manera que la Microbiología, la Bioquímica y la Entomología, son ofrecidas por diversas instituciones nacionales.

Pero este proceso no se ha confinado solamente a las Ciencias Naturales o a las disciplinas aplicadas. También ha aparecido en las Ciencias Humanas: en Economía y en Historia tanto como en Filosofía, en Sicología y en las facultades de Educación. En unas y otras han surgido posgrados para formar recursos humanos demandados por la modernización del Estado que tuvo lugar durante el Frente Nacional. Aparecieron los técnicos en planificación, los investigadores y los especialistas en administración, consejería y supervisión escolar, lo mismo que una intelligentsia interesada en el desarrollo de la cultura y de las ciencias en el país.

Los posgrados, sin embargo, no se expandieron solamente corno respuesta a procesos técnicos o a demandas ocupacionales provocadas por cambios económicos y sociales. Ellos son también producto de las presiones de los egresados de la universidad, quienes buscan más educación con la finalidad de acceder a mayores ingresos, a ocupaciones de más prestigio, a un ascenso dentro de su profesión (como ocurre en el magisterio) o simplemente como defensa ante el creciente desempleo de personal calificado. Para ellos es claro que la explosión universitaria hizo más difícil la inserción ocupacional y que los requisitos educativos para determinados puestos se han vuelto también mayores. Si en el pasado reciente el ingreso a la base del profesorado universitario tenía como requisito la

\footnotetext{
* Sociólogo. Profesor U.P.N.

Digitalizado por RED ACADEMICA
} 
licenciatura, y en algunos casos ni siquiera esto, en la actualidad se exige el título de master o de doctorado. Estos reclamos de mayor educación para desempeñar una actividad que apenas ha cambiado internamente, comienzan a generalizarse en el mundo de la administración pública y privada y en todo el sistema ocupacional que requiere de alguna habilidad, por mínima que ella sea.

Las estadísticas de posgrado son todavía muy escasas e inseguras. De todas formas las más confiables son las de la División de Formación Avanzada del ICFES para el año de 1983 que se reproducen en el Cuadro No. 1. De los 361 programas que allí se registran, 195 tienen el rango de "Especialización", 160 de "Maestría" y 6 de "Doctorado". Si la universidad colombiana es un fenómeno eminentemente urbano, los posgrados acentúan esta característica, hasta el extremo. La sola ciudad capital, Bogotá, ofrece 206, que sumados a los de Cali y Medellín alcanzan un total de 300 programas, esto es, el $83 \%$ de la educación posgraduada del país. La División de Formación Avanzada, la oficina encargada de la supervisión de los posgrados en Colombia, no dispone de datos de matrícula, pero las estadísticas periódicas de educación superior del ICFES suministran alguna información que puede servir de orientación. En 1971 aparecen 1.530 estudiantes, número que había ascendido a 2.501 en 1976 y a 4.777 en 1981. No obstante lo incompletos que puedan ser estos datos, ellos evidencian un crecimiento acelerado de la población interesada en programas de graduados, que a juzgar por el pasado inmediato será mucho mayor en los próximos años ${ }^{90}$

\section{CUADRO No. 1 \\ POSGRADOS EN EL PAIS SEGUN AREA DEL CONOCIMIENTO}

\begin{tabular}{lcc}
\hline \multicolumn{1}{c}{ Área del conocimiento } & $\begin{array}{c}\text { No. de } \\
\text { programas }\end{array}$ & $\%$ | \\
\hline Ciencias de la salud & 169 & 47.0 \\
Economía y afines & 43 & 12.0 \\
Ciencias sociales, Derecho y Ciencias & 40 & 11.0 \\
Políticas & 32 & 9.0 \\
Ingeniería, Arquitectura y Urbanismo & 30 & 8.0 \\
Ciencias de la educación & 24 & 7.0 \\
Matemáticas y Ciencias Naturales & 12 & 3.0 \\
Humanidades y Ciencias Religiosas & 11 & 3.0 \\
Agronomía, Veterinaria y afines & 361 & 100.0
\end{tabular}

FUENTE: Estadísticas suministradas por la División de Formación Avanzada. ICFES, mayo 31 de 1983.

\footnotetext{
${ }^{90}$ Ver, ICFES, Estadísticas de la educación superior de los años 1971 a 1981. Por supuesto, estas estadísticas sólo registran la información de las instituciones colombianas. Ello no debe llevar, sin embargo, a olvidar que la población que ha adquirido títulos de posgrado en el exterior, muy difícil de cuantificar, ha jugado un papel importante en la orientación de los programas nacionales. En algunos casos ellos fueron sus fundadores y por algún tiempo sus únicos profesores.
} 
Dentro de la evolución general de los posgrados, los programas en educación han tenido sus propias acentuaciones. Comenzaron a manifestarse en la década del 60 pero sólo vinieron a afirmarse en el decenio siguiente como respuesta a los problemas creados por la expansión del sistema educativo en sus niveles primario, secundario y universitario. Si entre 1960 y 1975 la enseñanza primaria pasó de 1.690.361 alumnos a casi 4.000.000, la secundaria lo hizo de 243.226 estudiantes a cerca de 1.400.000. Esto ocasiono cambios en la organización y en la supervisión de las actividades educativas; las instituciones crecieron y su administración se hizo más compleja. En el interior de muchas concentraciones escolares, de universidades y de establecimientos de gran tamaño, como los INEM (Institutos Nacionales de Enseñanza Media Diversificada), fue surgiendo un orden burocrático integrado por directores, subdirectores, jefes de departamento, consejeros, orientadores, etc., cuyo objetivo era hacer más eficiente el proceso educativo. Antes de 1970 este cuerpo administrativo no existía en las instituciones colombianas, salvo en unas pocas universidades, donde por lo demás nunca llegó a tener la magnitud ni la especificidad actuales. Sólo ahora surgía con toda su fuerza anunciando la masificación de la educación nacional. Con ello el sistema educativo se veía también encadenado al "irresistible" proceso de burocratización weberiano que ya era corriente tanto en el aparato del Estado como en el mundo de la empresa, del comercio y de los negocios $^{91}$.

La Universidad Pedagógica Nacional jugó un papel importante en la consolidación de los estudios de posgrado en educación en el país. Con la participación de la UNESCO, inició actividades en 1971 en Administración Educativa, en Consejería Escolar, en Supervisión y en Sicopedagógica, programas por los cuales desfilaron varios centenares de estudiantes que después fueron reclutados por las secretarías departamentales de Educación, las escuelas normales, los INEM, las universidades y el Ministerio de Educación. Este boom se agotó en 1974 cuando las posibilidades ocupacionales se hicieron difíciles en estas entidades, y, si algunos de aquellos programas continuaron existiendo hasta finales de la década del 70, el tamaño de su matrícula disminuyó bruscamente y la relación positiva entre posgrado y éxito ocupacional comenzó a visualizarse como una aspiración cuya realización era cada vez más remota.

Ante esta situación la Universidad Pedagógica Nacional se interesó por nuevas especialidades no explotadas hasta el momento, como la investigación educativa y el análisis curricular ${ }^{92}$. En realidad, este interés había sido postulado con anterioridad por diversas agencias del Estado, las cuales habían hablado ya en varias ocasiones de la necesidad de formación de personal calificado en los campos de la investigación y del currículo. Su formulación más clara se encuentra en el programa de desarrollo de la administración López Michelsen (1974-1978) conocido con el nombre de Para cerrar la brecha, donde abiertamente se consignó que uno de los problemas que más afectan al sistema educativo es,

\footnotetext{
${ }^{91}$ Aunque es difícil de evaluar su monto, la expansión educativa ocasionó otras fuerzas que contribuyeron al crecimiento de los posgrados en educación. El aumento mismo del grupo docente exigió una regulación del magisterio adecuada a las nuevas situaciones. Así, al promulgaras en 1979 el Estatuto Docente - la reglamentación de la profesión de maestro primario y secundario por parte del Estado-, se estipuló que los ascensos dentro del oficio se podían alcanzar no solamente a través de la edad o de los años de servicio, sino también por la producción intelectual y por la posesión de títulos de nivel graduado. Ello hizo que los maestros más jóvenes en ejercicio con título universitario, especialmente los de los centros urbanos, comenzaran a tocar las puertas de los posgrados y a presionar a las universidades y a las facultades de Educación por programas de graduados. En otras palabras, los posgrados en educación se convirtieron para algunos de sus moradores en un mecanismo de movilidad dentro de un esquema institucional previamente establecido.

${ }^{92}$ Algún tiempo después abrió posgrados en Enseñanza de la Física, de la Matemática, de la Salud y de la Docencia Universitaria. Ello quiere decir, que de los 30 programas en educación registrados en el Cuadro No. 1, la tercera parte de ellos pertenecía a la Universidad Pedagógica Nacional.
} 
"La carencia de investigación básica y aplicada, particularmente de investigación socioeducativa y curricular, trae como consecuencia el desconocimiento de las características culturales y, por lo tanto, contenidos curriculares y métodos de enseñanza inadecuados para las necesidades de cada región. Generalmente se ha recurrido a la copia indiscriminada de métodos y técnicas de educación de otros países. Además, la ausencia, de una evaluación del rendimiento interno y externo del sistema ha privado al país de experiencias valiosas en el campo de la enseñanza" ${ }^{93}$.

Esta fue la génesis de los posgrados de Investigación Socioeducativa y de Análisis Curricular de la Universidad Pedagógica Nacional. El primero habría de tener una orientación sociológica y el segundo una sicológica, con énfasis en la tradición teórica vinculada al análisis de la conducta (behaviorism). Si en un principio estuvieron estrechamente asociados, especialmente en los primeros semestres donde compartieron las asignaturas de metodología y estadística, sus intereses temáticos y sus perspectivas teóricas los fueron separando hasta hacerse entidades autónomas y completamente independientes.

El posgrado de investigación Socioeducativa fue el primer esfuerzo institucional emprendido en el país por ofrecer una respuesta coherente y sistemática a las demandas de personal calificado en los aspectos sociales de la educación. Antes de su creación, la formación de investigadores se realizaba generalmente en instituciones extranjeras, en las cuales se daba preferencia a modalidades y contenidos no siempre relevantes para los problemas nacionales. Además, los posgrados fundados a comienzos de la década del 70 estaban, como se ha visto, enfocados hacia el entrenamiento de recursos humanos en tareas aplicadas como la administración, la consejería o la supervisi4n escolares. Si bien cada uno de estos campos exige alguna familiaridad con los métodos y las técnicas de investigación, su objeto central es la atención y la vigilancia del normal desarrollo de las labores de la enseñanza. Su función es resolver los múltiples problemas que presenta la dinámica interna del sistema educativo. Sus oficiantes pueden hacer uso de los resultados de investigación, pero no tienen el tiempo ni el adiestramiento necesarios para adelantarla.

El presente trabajo busca evaluar el camino seguido por el programa de Investigación Socioeducativa, el único posgrado en ciencias sociales de la Universidad Pedagógica Nacional. En las primeras secciones se describen sus cambios curriculares y las características de sus profesores y de sus estudiantes, para pasar después a una discusión de la inserción ocupacional de sus egresados, y terminar con una estrategia curricular que recoge las experiencias adquiridas a lo largo de ocho años de actividad ininterrumpida (1975-1983). La exposición tiene un tono analítico con la finalidad de facilitar la derivación de elementos generales a partir de la exposición de un caso particular. Ante la ausencia de investigaciones en este campo, se espera que el trabajo

\footnotetext{
${ }^{93}$ Departamento Nacional de Planeación, Para cerrar la brecha (Bogotá: Ediciones del Banco de la República, 1975), p. 154. Este interés del Estado por la investigación socioeducativa fue constante durante la segunda mitad de la década del 70 y comienzos de la del 80. Así, el Plan de Integración Nacional (PIN), el programa de desarrollo de la administración Turbay Ayala (1978-1982), afirmó la necesidad de "la investigación socioeducativa como fundamento de las acciones que se desarrollen en el sector". El Pían.., buscaba que la reforma de la enseñanza partiera de un conocimiento de las condiciones económicas, sociales y culturales de la población, con el fin de experimentar y adecuar los contenidos y los métodos educativos "a las condiciones de las diferentes regiones y grupos sociales". Ver Departamento Nacional de Planeación, Plan de Integración Nacional (Bogotá: Industria Continental Gráfica, 1980), Tomo I, p. 226.
}

La misma idea vuelve a ser ratificada por el plan de desarrollo del Presidente Belisario Betancur, en el cual se subraya que "la mejora de la calidad de la educación dependerá del perfeccionamiento de la investigación sobre las características sociales y económicas de la enseñanza en Colombia”. (Ver Departamento Nacional de Planeación, Cambio con equidad: 1983-1 986 (Bogotá: Canal RamírezAntares, 1983), p. 283. 
contribuya de alguna manera a promover evaluaciones de otros programas con el fin de adquirir un conocimiento más acabado de los resultados de la educación posgraduada, una parte del sistema educativo ahora en franca expansión en el país.

\section{Objetivos del programa y estructura académica}

El posgrado de Investigación Socioeducativa abrió sus puertas en septiembre de 1975. Durante sus primeros años se planteó dos objetivos básicos: entrenar profesores de metodología para las facultades de Educación -las instituciones encargadas del adiestramiento de los docentes que estarían al frente de la expansión educativa-, y formar investigadores con especial sensibilidad en las dimensiones sociales de los procesos educativos. Estos objetivos eran complementarios. Se esperaba que sus egresados adelantaran tareas de investigación y que además desarrollaran una labor multiplicadora en las universidades comprometidas en la formación de maestros. Ambos objetivos eran una respuesta a las demandas de investigación socioeducativa expresadas una y otra vez por el Estado a través de sus planes nacionales de desarrollo ${ }^{94}$.

Para llevar a cabo estos objetivos se diseñó un currículo dividido en dos partes: cursos básicos y cursos de especialización. Los básicos estaban dirigidos a dar una información general sobre educación y sobre los problemas lógicos de la investigación científica. Las asignaturas que expresaban estos fines eran:

- Sociología de la educación

- Teoría general de la educación

-Filosofía y lógica de las ciencias

—Métodos y técnicas de investigación

Los cursos de especialización estaban orientados a desarrollar destrezas en campos especiales del conocimiento y a profundizar las temáticas esbozadas en la primera parte. Con ellos se buscaba proporcionar los marcos de referencia y los instrumentos teóricos necesarios para emprender investigaciones en educación. Sus asignaturas eran:

- Economía de la Educación

- Estructura y procesos sociales (sociología del desarrollo)

- Análisis demográfico aplicado a la educación

- Planificación y evaluación de programas educativos

- Seminarios de sociología de la educación: Durkheim y Weber

- Metodología avanzada (diseños experimentales, modelos matemáticos aplicados a la educación, análisis multivariado, análisis de varianza y análisis causal con datos no experimentales) ${ }^{95}$.

Este conjunto de asignaturas estaba distribuido en cuatro períodos que sumaban cerca de dos años de labor académica (los cursos básicos estaban concentrados en los dos primeros y los especializados en los últimos). Las actividades rutinarias giraban alrededor

\footnotetext{
${ }^{94}$ Durante los primeros años el posgrado contó con la ayuda del Proyecto Col. PNUD-UNESCO 72/031, con sede en el antiguo ICOLPE (Instituto Colombiano de Pedagogía). Entre sus expertos, Guillermo Briones fue el más entusiasta promotor del programa y por varios semestres estuvo encargado de su sección de Metodología. Briones elaboró el proyecto que dio lugar al posgrado y redactó el primer plan de estudios alrededor del cual se organizaron las actividades académicas. A través de la UNESCO se canalizaron también auxilios para expandir los recursos bibliográficos y se contó con la consejería de sus expertos para los necesarios ajustes curriculares de todo plan que apenas comenzaba a dar sus primeros pasos.

${ }^{95}$ Ver, Universidad Pedagógica Nacional, Anteproyecto: Programa de magíster en educación con especialidad en investigación (Bogotá, junio de 1975), pp. 3 y 4. Dos asignaturas que aparecen en este anteproyecto., “Análisis de sistemas" y "Metodología de la educación a distancia", no se ofrecieron por carencia de profesores especializados.
} 
de las conferencias de los profesores y de los seminarios donde los estudiantes tenían una amplia participación, y en los cuales se esperaba que los alumnos comenzaran a familiarizarse con un problema de investigación que los condujera directamente a su tesis de grado.

Pero como ocurre en las etapas iniciales de todo proyecto universitario, este currículo fue reorganizado una vez que la primera generación de estudiantes terminó sus tareas. Para ese momento era evidente que el posgrado carecía de una idea matriz que orientara y diera vida a sus actividades. No había una perspectiva que agrupara las diversas asignaturas y cada una de ellas parecía tener sus propios fines. Ello se debía a la ausencia de una estrategia teórica que integrara la marcha del programa en relación con los objetivos previamente diseñados. Esta estrategia se halló en la sociología de la educación, esto es, en la postulación de las interacciones entre educación y sociedad como marco general. Se afirmó que toda acción educativa tiene lugar en un escenario social, cuya función es expresarlo, reproducirlo y ei algunos casos contribuir a su transformación. Así, a partir de la experiencia integradora de una ciencia, en este caso de la Sociología, se encontró un terreno común para unificar las disciplinas y las labores de posgrado.

Esto produjo algunos cambios que se produjeron en una definición más clara de las metas académicas y en una reorganización de las asignaturas, cambios que fueron recogidos en el Prospecto de 1977, cuando finalizaba el segundo año de vida del posgrado. Con un currículo más orgánico, se buscaba desarrollar en los estudiantes las siguientes destrezas: ${ }^{96}$

a. Manejo de los métodos y técnicas de investigación aplicadas a la educacion.

b. Habilidad en la construcción de teorías y comprobación o rechazo dc hipótesis.

c. Capacidad analítica para la presentación y desarrollo de diseños de investigación, y

\section{d. Aptitud en la elaboración y seguimiento de proyectos de planeación educativa.}

Los objetivos generales del programa, que de hecho se confundían con las posibilidades ocupacionales de sus egresados, también sufrieron una ampliación. Su mercado de trabajo sería el mundo universitario, dentro del cual las facultades de Educación seguían ocupando un papel importante, y los centros de investigación oficiales y privados interesados en la investigación educativa. A ello se sumaba el fortalecimiento de las oficinas de planeación donde la formulación y evaluación de políticas educativas se había convertido en una tarea prioritaria.

El plan de estudios que expresaba estos cambios estaba ahora dividido en tres áreas estrechamente relacionadas: área de teoría social, de metodología y de asignaturas de carácter interdisciplinario. La primera ofrecía las herramientas conceptuales para la crítica y el análisis de datos y para la elaboración de marcos de referencia acordes con los problemas del medio. Los cursos que llenaban esta finalidad eran: Sociología de la educación, Seminario de sociología de la educación (Durkheim y Weber) y Estructura y procesos sociales. La segunda área tenía por objeto desarrollar habilidades en el manejo de los métodos y de las técnicas de investigación aplicadas a la educación. Incluía

\footnotetext{
${ }^{96}$ Universidad Pedagógica Nacional, Programa de posgrado en investigación socioeducativa (Bogotá: 1977), pp. 2 y 3 . Una segunda edición de este Prospecto se publicó en 1978.
} 
además una reflexión sobre las características del método científico y una discusión de la lógica de la investigación social. Sus cursos eran: Lógica de la ciencia, Métodos y técnicas de investigación (I y II), Seminario de tesis y Estadística. La tercera área buscaba integrar los hallazgos de diversas disciplinas que abordan la educación desde una perspectiva social. Sus asignaturas eran: Economía de la educación, Demografía y Educación, Teoría de la educación y Planeamiento Educativo ${ }^{97}$.

Las materias de estas áreas fueron distribuidas en los cuatro períodos académicos como sigue: ${ }^{98}$

\section{Primer período}

Sociología de la educación

Lógica de las ciencias.

Teoría general de la educación

Estadística (1)

\section{Segundo período}

Seminario de sociología de la educación

(Durkheim y Weber).

Estructura y procesos sociales

Métodos y técnicas de investigación (I)

\section{Tercer Período}

Economía de la educación

Métodos y técnicas de investigación (II)

Estadística (II)

\section{Cuarto período}

Seminario de tesis

Planeamiento educativo

Demografía y educación

Esta habría de ser la estructura curricular del posgrado hasta nuestros días. Los cambios que se hicieron en los años siguientes fueron solamente de ajuste, de remplazo de unas materias por otras o de redefinición del contenido de algunas de ellas.

Pero la mayor innovación que acompañaba todas estas reformas, y que apenas se manifiesta en una lectura del nuevo plan de estudios, era la relación entre teoría e investigación, asunto que dio lugar a continuas discusiones en el seno del posgrado. Desde un comienzo se quiso afirmar el principio de que una investigación que no esté basada en un permanente intercambio entre teoría y hechos, entre datos y marcos de referencia, conduce a un resultado bastante pobre. Partiendo de esta idea se quiso diseñar un programa donde la formación teórica y el desarrollo de las habilidades para el manejo y el tratamiento de datos fueran considerados como partes de una misma empresa. Este interjuego llevaba también la preocupación de que la única manera de sacar la investigación educativa de las pasivas e ingenuas descripciones asociadas a la lectura de estadísticas oficiales, era la asimilación de la teoría ofrecida por lo mejor de la historia del pensamiento social.

Esta fue la puerta de entrada de los "Seminarios de sociología de la educación", a través de los cuales se buscaba una familiarización con las obras de algunos de los

\footnotetext{
${ }^{97}$ Aunque era evidente que en esta área debía incluirse la Historia y la Antropología de la educación, muy desarrolladas en otros países, no se lo hizo en aquella época por carencia de profesores idóneos. La Historia apenas comenzaba a explotarse como campo fructífero en educación y la Antropología sólo presentaba ejemplos dispersos y escasos que no lograban conformar un cuerpo de alguna coherencia. Varios egresados del posgrado irían a trabajar tiempo después con éxito en estos campos.

${ }^{98}$ Universidad Pedagógica Nacional, Programa de posgrado en investigación socioeducativa (Bogotá: 1977), pp. 7-9 y 11-12.
} 
grandes pensadores que hoy llamamos los clásicos de la ciencia social ${ }^{99}$. Con este contacto se esperaban promover los problemas de mayor significación en la investigación educativa, además de desarrollar la capacidad analítica y la imaginación teórica de los jóvenes investigadores. Al principio se experimentó con un seminario sobre la obra de Emilio Durkheims y de Max Weber, especialmente en lo relacionado con su tratamiento de los fenómenos educativos, y ante su éxito, en 1979 se introdujo otro dedicado al estudio de las contribuciones de Karl Mannheim, figura que había ampliado las discusiones educativas iniciadas por aquellos pensadores ${ }^{100}$. A este respecto cabe anotar que el posgrado de Investigación Socioeducativa ha sido el único programa de graduados en el país que ha intentado abordar en forma sistemática el estudio de los clásicos en un campo determinado del conocimiento - la sociología de la educación. Esta ha sido sin duda una innovación en la Universidad Pedagógica Nacional en particular y en las facultades de Educación en general, donde tradicionalmente la enseñanza se ha hecho a través de manuales, de textos introductorios o de resúmenes de las tradiciones teóricas de una disciplina. En ellas no ha existido contacto alguno con los grandes pensadores ni una discusión de sus principales hipótesis a partir de una familiarización con los textos originales.

Con estas aspiraciones se puso en marcha el posgrado de Investigación Socioeducativa de la Universidad Pedagógica Nacional. Sus realizaciones, sus resultados y experiencias serán objeto de estudio en las páginas siguientes.

\section{Profesores y estudiantes}

Desde su fundación hasta el presente, excepto para el año académico de 1981-1982 cuando el educador Jorge Murcia Florián estuvo encargado de su dirección, el posgrado ha sido coordinado por el sociólogo Gonzalo Cataño. Cataño ha sido además el único profesor permanente del programa financiado por la Universidad Pedagógica Nacional. En sus ocho años de vida el posgrado sólo ha contado con la ayuda de dos docentes de tiempo completo y ambos por períodos cortos. El primero de ellos fue el profesor Mauricio Ferro Calvo, quien trabajó durante tres años entre 1976 y 1978, y el segundo, el sociólogo Fernando Uricoechea, quien colaboró con las actividades del programa entre 1980 y 1982.

Ante la ausencia de profesores de tiempo completo, se optó por cubrir las obligaciones académicas con docentes invitados. Su selección fue bastante rigurosa durante los primeros seis años y por aquella época se contó con la ayuda de varios líderes en diversos campos de las Ciencias Humanas del país ${ }^{101}$. Estos profesores desencadenaron temas de investigación, lecturas e inquietudes intelectuales entre los estudiantes y le confirieron al pos-grado un prestigio externo que se reflejó en las múltiples invitaciones que recibió para colaborar en los encuentros educativos y en las discusiones de investigación que se realizaron a finales de los años setenta. Aquella fue también la época

\footnotetext{
${ }^{99}$ Se entiende por clásicos — Freud en sicología; Smith, Ricardo y Keynes en economía; Durkheim, Weber y Simmel en sociología, etc.- , aquellos pensadores que han logrado delimitar un campo de investigación o que han conseguido formular un marco de referencia que se convierte en paradigma y orientación permanentes para los futuros investigadores del área.

${ }^{100}$ Además del seminario de sociología de la educación dedicado a Mannheim, por esta fecha se introdujo también el curso de "Historia de la Educación Colombiana", una vieja aspiración que como se sabe estaba implícita en los objetivos del posgrado. Al introducir estos dos nuevos cursos, se dejaron de ofrecer los de "Planeamiento educativo" y "Demografía y educación, cuyos contenidos más relievantes fueron asimilados por las clases de metodología (evaluación de programas) y de estadística (técnicas demográficas). En esta forma se evitó el aumento de asignaturas, peligro al que siempre está expuesto todo posgrado ante las ilimitadas posibilidades curriculares, lográndose así un plan de estudios mucho más acabado que los anteriores.

${ }^{101}$ Personas como Orlando Fals Borda, Carlos Escalante, Rubén Sierra Mejía, Bernardo Kugler y Mario Arrubla, ofrecieron en su orden cátedras de metodología, de técnicas de investigación, de lógica de las ciencias, de economía de la educación y de desarrollo económica y social. A ellos se sumaron profesores más jóvenes que se han destacado en sus especialidades, como Eduardo Vélez Bustillo (estadística), Carlos Becerra (diseño de encuestas), Gabriel Restrepo (Sociología de la ciencia), Humberto Rojas (Sociología aplicada) y Jesús Bejarano (desarrollo económico).
} 
de la creación de la Revista Colombiana de Educación en el Centro de Investigaciones de la Universidad Pedagógica Nacional (CIUP), en cuya fundación y promoción el posgrado participó activamente. Allí publicaron artículos y notas críticas varios de sus profesores, y sus estudiantes se convirtieron en los más asiduos consumidores y en los más entusiastas propagadores de la Revista ${ }^{102}$.

Pero una organización docente de estas características creó sus propios problemas. Al sustentar el grueso del currículo en profesores vinculados por unas pocas horas a la semana, produjo en los estudiantes unas demandas que la endeble estructura administrativa del posgrado nunca logró resolver satisfactoriamente. Si durante sus horas de clase eran objeto de una enseñanza enriquecedora, en sus horas de trabajo apenas tenían quien los orientara y les ofreciera una tutoría que diera vida a su aprendizaje teórico. Ello cobró más fuerza en el momento en que los estudiantes terminaban sus clases y debían enfrentarse con sus tesis de grado. Esta dinámica hizo que buena parte de las labores de orientación y de supervisión de los trabajos de investigación y de tesis descansara en última instancia en la frágil coordinación del posgrado. En otras palabras, la loable y positiva política de traer profesores de reconocido prestigio, fue neutralizada por la ausencia de una organización académica que tuviera la capacidad de canalizar las innovaciones promovidas por los docentes invitados. Ello generó un sentimiento de frustración en los estudiantes de mayor vocación intelectual e impidió la consolidación de un clima de investigación en el seno del posgrado.

En relación con los estudiantes, las experiencias del posgrado no han sido menos valiosas. Como toda escuela de graduados, el programa de Investigación Socioeducativa exigió desde un principio que sus aspirantes portaran un grado o título universitario otorgado por una universidad reconocida por el Estado. A este requisito general se sumaron otras exigencias más específicas dirigidas a cubrir los objetivos especiales del posgrado. En primer lugar, se buscó una afinidad entre los anteriores estudios y actividades del candidato con el contenido de los cursos a los cuales iba a estar expuesto durante dos años. En segundo lugar, se trató de evaluar su vocación intelectual a través de sus realizaciones académicas, su interés por la investigación, sus aspiraciones, sus anhelos y su capacidad analítica. $Y$ en tercer lugar, se indagó sobre las ayudas económicas para adelantar el pos-grado (posibilidades de beca, préstamos, licencia remunerada, recursos propios, etc.). Esta última demanda habría de ser al final muy frágil, a causa de las drásticas restricciones presupuéstales del Estado y de las universidades públicas y privadas en materia de becas y de licencias remuneradas para sus funcionarios y profesores. Ello hizo que en la práctica las dos terceras partes de los estudiantes combinaran sus estudios graduados con jornadas laborales de medio tiempo o de tiempo completo. Esto ocurrió no sólo con los estudiantes que venían de otras regiones del país, sino con los mismos profesores de pregrado de la Universidad Pedagógica Nacional que deseaban adelantar sus estudios de posgrado en una especialidad ofrecida por su Universidad. Así, los posgrados de Administración, de Consejería Escolar o de Investigación Curricular fueron realizados por muchos profesores de la Universidad sin interrumpir sus obligaciones docentes o administrativas dentro de la institución.

Esta situación ha repercutido, por supuesto, en la calidad del posgrado. Si de alguna manera los estudiantes han cumplido con las clases y con las exigencias vinculadas a las asignaturas teóricas, es poco lo que consiguen en el terreno de la investigación y de sus

\footnotetext{
${ }^{102}$ La Primera entrega de la revista apareció en jumo de 1978 y en este momento cuenta con 12 números en el mercado. En su estudio, Informe sobre la evaluación del programa de maestría en investigación socioeducativa (Bogotá: U.P.N., 1982), pp. 33 y 34 , Jorge Murcia Florián menciona algunas de las publicaciones de los profesores aparecidas en la revista.
} 
trabajos de tesis. En relación con esto último, puede afirmarse que la baja frecuencia de graduados en los programas de la Universidad en general, y en el de Socioeducativa en particular, se debe a que las obligaciones laborales de sus egresados no les permite el tiempo y el reposo necesarios para adelantar sus trabajos de tesis ${ }^{103}$.

Todo esto viene entonces a robustecer las limitaciones derivadas de la estructura docente del posgrado. Hay, por un lado, escasez de profesores de tiempo completo para atender a los estudiantes, y, por el otro, éstos apenas disponen de tiempo para sus labores de investigación y sus tesis de grado.

Para facilitar las actividades docentes, desde un comienzo se estableció como política del posgrado admitir pequeños grupos de estudiantes, y sólo cuando una generación llegaba a su fin se hacía la convocatoria para la siguiente ${ }^{104}$. Así se evitaron las aglomeraciones, típicas en los demás posgrados de la Universidad, y se obviaron las posibles saturaciones del mercado de trabajo especializado en investigación y docencia y en diseño y evaluación de políticas educativas. Las estadísticas de las diversas generaciones del posgrado aparecen en el cuadro siguiente:

CUADRO No. 2

\section{ESTUDIANTES DEL POSGRADO DE INVESTIGACION SOCIO-EDUCATIVA}

\begin{tabular}{ccc}
\hline Años & $\begin{array}{c}\text { No. de } \\
\text { estudiantes } \\
\text { matriculados }\end{array}$ & $\begin{array}{c}\text { No. de estudiantes } \\
\text { que terminaron } \\
\text { satisfactoriamente }\end{array}$ \\
\hline $1975-1977$ & 18 & 12 \\
$1977-1978$ & 12 & 11 \\
$1979-1980$ & 21 & 11 \\
$1981-1982$ & 9 & 8 \\
$1982-1983$ & 7 & 42
\end{tabular}

FUENTE: Archivos de matrícula de la Universidad Pedagógica Nacional compilados por Jorge Murcia Florián en su Informe sobre la evaluación del programa de maestría de investigación socio-educativa (Bogotá: Universidad Pedagógica Nacional, 1982), p. 75.

La primera columna muestra los estudiantes que se han matriculado en cada una de las promociones (generaciones) del posgrado, y la segunda, los estudiantes que han terminado satisfactoriamente sus cursos. Cerca de la mitad de los 25 estudiantes que han interrumpido sus estudios en diversos momentos, esperan concluir sus cursos en un

\footnotetext{
${ }^{103}$ Esta es por lo demás la situación de la mayoría de los posgrados en el país, tanto en ciencias sociales como en ciencias naturales y exactas. Al reclutar profesionales que están insertos en la estructura ocupacional, no se han ideado estrategias para hacer que estos puedan desarrollar sus tesis en forma paralela a sus obligaciones laborales.

${ }^{104}$ Esta sana política no fue observada en 1982, año en el cual se hizo una convocatoria bastante improvisada que tuvo consecuencias negativas para el posgrado. Se presentaron muy pocos aspirantes y no se planeé con suficiente antelación el grupo de profesores que había de estar al frente de las asignaturas. En ese año se admitieron siete estudiantes y al poco tiempo habían desertado dos de ellos por diversas razones.
} 
futuro cercano -y varios de ellos han tomado ya algunas materias. La generación que comenzó en febrero de 1982, cursa ahora su cuarto semestre. Además de esta matrícula regular, el posgrado ha contado a lo largo de sus años con un volumen amplio de alumnos de otros programas de la Universidad, entre 25 y 30 , que han tomado diversas clases de Investigación Socioeducativa con el objeto de completar sus créditos o de llenar sus vacíos en ciencias sociales.

Hasta el momento se han graduado 10 estudiantes con las siguientes tesis ${ }^{105}$

Diana E. Soto Arango: "La educación superior y el desarrollo económico en el Departamento de Córdoba” (Bogotá: U.P.N., 1978).

Fidel Salazar Reyes: "Estudiantes y machismo: opinión de jóvenes de enseñanza media" (Bogotá: U.P.N., 1980).

Ernesto Botello Pérez: “Educación y desarrollo en Panamá: 1960-1980" (Bogotá: U.P.N., 1981).

Alberto Martínez Boom: "De la evangelización a la instrucción pública: contribución al estudio de las prácticas educativas coloniales" (Bogotá: U.P.N., 1981).

Milciades Vizcaíno Gutiérrez y Julio E. Díaz Beltrán: “Televisión y educación de adultos en Colombia" (Bogotá: U.P.N., 1982).

María C. Araoz: "Investigación evaluativa sobre los programas de internado en el SENA" (Bogotá: U.P.N., 1983).

Jean-Claude Gerlus: "Las tendencias de la educación superior en Haití: realidades actuales y perspectivas futuras" (Bogotá: U.P.N., 1983).

Beatriz Céspedes: "Desempeño ocupacional, educativo y social de los bachilleres industriales" (Bogotá: U.P.N., 1983).

Marcos González: "Francisco José de Caldas y la ilustración de la Nueva Granada" (Bogotá: U.P.N., 1983).

Ante el escaso número de graduados, la coordinación del posgrado ha desarrollado una ofensiva dirigida a promover la presentación de proyectos y a impulsar los que se venían adelantando en el pasado. Esta política ha dado excelentes resultados y en este momento hay 9 tesis en marcha. Ellas son:

Jorge E. Rozo y Oscar Jaimes: “El Gimnasio Moderno: historia y situación actual”.

Cecilia Herrerías de Sarabia: "Políticas y realidades de la educación de adultos en Colombia: una década de expectativas, 1970-1979".

María del Carmen Quezada: "Educación y migración: el caso de Villeta (Cundinamarca).

\footnotetext{
${ }^{105}$ Clemencia Leyva Franco, Índice de tesis de grado (Bogotá: Universidad Pedagógica Nacional Centro de Documentación Educativa, 1982), passim.
} 
Rodolfo Posada: "La ilustración europea y su influencia en el plan educativo de Moreno y Escandón (1774)".

Miguel Borja: "Aspectos cuantitativos de la educación colombiana durante el S. XIX".

Jaír Duque y Mamela Prieto: “Las escuelas lancasterianas en Colombia: 1824-1845”.

Yolanda Ramírez Prado: traducción de Max Weber On Universities (con una introducción de la traductora).

Leonor Gómez G. y Luis Lara: "Situación actual de la supervisión escolar en Colombia”.

Leonor Zubieta: “Las escuelas normales: un estudio etnográfico”.

Cerca de las dos terceras partes de los estudiantes provienen de facultades de Educación. El resto está conformado por egresados de filosofía, sociología, trabajo social y demás especialidades de las ciencias humanas. El hecho de que el grueso de la matrícula provenga de los medios pedagógicos, ha influido para que el posgrado invierta parte de sus esfuerzos en una enseñanza introductoria en materias tales como metodología, estadística, sociología y economía, cursos que apenas existen en las facultades de Educación. Esto se ha convertido muchas veces en un freno, si no en un obstáculo, para ofrecer las asignaturas de mayor complejidad demandadas por los estudiantes de más expectativas intelectuales. Así, el impacto de los seminarios y de la lectura de ciertos autores y tradiciones de pensamiento, uno de los rasgos más innovadores del posgrado según se apuntó en páginas anteriores, se ha visto disminuido por las características formativas de la mayoría de sus estudiantes - que al final imponen el clima y el ritmo de todo programa académico-.

Todo esto produjo tensiones internas que alimentaron una ambivalencia que ha sido característica del posgrado de Investigación Socioeducativa. Por un lado, ha cumplido la función de transmitir una información básica en materia de investigación a un grupo de personas que carecía de ella -y este ha sido uno de los aspectos más positivos del posgrado-, y por el otro, ha frustrado a aquellos estudiantes que buscaban una enseñanza más compleja y operativa, especialmente referida a las prácticas de investigación y al uso de teorías más sofisticadas.

Con el objeto de encontrar una respuesta a esta situación, la convocatoria de 1981 subrayó que el posgrado estaba "diseñado para aquellos aspirantes con antecedentes en ciencias sociales (antropólogos, historiadores, sociólogos, etc.) que estén interesados en los problemas educativos, y para aquellas personas que estén actualmente vinculadas a labores de investigación y planeamiento en el campo de la educación"106 - Con esto se buscaba un cambio en los antecedentes formativos de los candidatos y se pretendía encontrar un grupo estudiantil más afín a la investigación y al desarrollo teórico del programa. Sin embargo, el éxito fue parcial por la escasa presencia académica de la Universidad Pedagógica Nacional entre la población que no ha transitado previamente por una institución de carácter pedagógico. Se encontró que ella no poseía legitimidad intelectual ante los egresados de otras carreras universitarias. Su limitada creatividad y sus énfasis en los aspectos estrictamente operativos de la educación —problemas de aprendizaje, métodos y técnicas vinculados a la transmisión del conocimiento, administración y consejería escolar, etc-, hacían que ella no tuviera ninguna visibilidad

\footnotetext{
${ }^{106}$ Universidad Pedagógica Nacional, Programa de posgrado en investigación socioeducativa (Bogotá: 1981), p. 3.
} 
para personas con vocación intelectual o con un entrenamiento en una disciplina científica particular. Esta característica la comparte por supuesto con las demás instituciones pedagógicas, las facultades de Educación y las escuelas normales, donde generalmente se transmite un conocimiento de compendio y resumen que después es difundido por sus egresados en las escuelas primarias y en los colegios de secundaria. A esto se suma el bajo prestigio de las facultades de Educación que, como se sabe, entrenan para una ocupación dependiente, asalariada y de escasa remuneración dentro del ámbito de las clases medias.

Esto muestra que la incorporación de un clima afín a la investigación y a la creatividad intelectual en los centros dedicados a la formación de docentes, es un proceso mucho más complejo del que generalmente presentan los planificadores de la educación. Estos centros exhiben una cultura y una dinámica interna que neutralizan las innovaciones que se introducen en sus claustros. Su matrícula estudiantil — tanto de pregrado como de posgrado-, es "endogámica,', esto es, proviene en su mayoría de los medios pedagógicos y busca recrear en los estudios graduados la experiencia adquirida en el pregrado. Esta dinámica institucional se convierte entonces en un obstáculo para el normal desarrollo de los procesos de modernización, y al final resulta deformando los objetivos de los programas que intentan transforma su tradicional estilo de trabajo académico $^{107}$.

\section{Opiniones sobre el programa}

En 1982 el profesor Jorge Murcia Florián de la Facultad de Educación de la Universidad Pedagógica Nacional, realizó una evaluación del posgrado de Investigación Socioeducativa. El autor efectuó entrevistas con estudiantes, egresados y especialistas en educación —del ICFES y de la Universidad de Antioquia, donde existe un programa similar-, sobre los objetivos del posgrado y sobre su utilidad e importancia.

La información ofrecida por el trabajo de Murcia Florián debe entenderse como un mero sondeo de opinión y no como el fruto de una investigación rigurosa: El universo de estudiantes en 1982 era tan reducido, 16 estudiantes solamente, que a partir de él no puede extraerse muestra alguna como lo pretende el autor. Lo mismo ocurre con los egresados. De 34 alumnos que en 1982 habían terminado sus estudios graduados, sólo 5 retornaron el cuestionario que previamente se les había enviado. Murcia Florián apunta que ante esta situación adelantó entrevistas personales con otros egresados, "informantes claves (y) de fácil acceso", con el objeto de alcanzar una información más coherente ${ }^{108}$. Sin embargo, el lector no encuentra noticia de su número a pesar de que la lectura de las respuestas se hace en porcentajes. Como se sabe, el uso de porcentajes tiene sus reglas --cuando el número de casos es muy pequeño su lectura debe hacerse en unidades y no en porcentajes. Por ejemplo ¿qué sentido tiene habla del 710/o de los estudiantes cuando se está trabajando con siete casos? A pesar de estas limitaciones metodológicas, el trabajo arroja algunos resultados sobre las experiencias del posgrado que proporcionan valiosas orientaciones para identificar problemas y diseñar políticas para el futuro.

\footnotetext{
${ }^{107}$ En buena parte de los posgrados en educación del país, a la "endogamia" estudiantil se suma el "incesto" académico, esto es, la presencia de un cuerpo docente egresado de la misma universidad y aún del mismo posgrado donde ahora enseñan. En estos programas estudiantes y profesores se convierten en un mecanismo de reproducción de los estilos de trabajo dominantes en la institución y en una muralla contra las "ideas extrañas" - las formas de trabajo de los demás centros universitarios-.

108 Jorge Muria Florián, informe sobre la evaluación del programa de maestría en investigación socioeducativa (Bogotá: Universidad Pedagógica Nacional, 1982), p. 49.
} 
Los diez estudiantes que en 1982 contestaron el formulario, observaron que su vinculación al posgrado se debía a su interés por "elevar el nivel de conocimientos" y a su especial inclinación por el campo ofrecido en el programa ${ }^{109}$. Pero también señalaron que los objetivos del programa no se habían logrado ni respondían a las necesidades del medio. Respecto a los profesores y a la organización del posgrado, consideraron que había numerosas limitaciones, como la "falta de estímulos para desarrollar actividades", la "insuficiente dotación de recursos didácticos", la "inadecuada metodología y dedicación del profesorado", y la "deficiente orientación y selección de los estudiantes". En relación con esto último, llama la atención la conciencia autocrítica de las personas que evaluaban sus propios estudios graduados. Manifestaron, así mismo, la ausencia de prácticas de investigación en su formación y destacaron las limitaciones institucionales en materia de biblioteca y de recursos bibliográficos especializados.

Pero no todas las respuestas portaban un tono negativo. A pesar de que los informantes se desempeñaban todavía como estudiantes, la mitad de ellos respondió que el posgrado había "contribuido al desarrollo de su profesión". En esta misma dirección, conceptuaron que la Metodología de la investigación, la Sociología de la educación y la Lógica de la ciencia, eran las asignaturas más importantes del programa y posiblemente las que más habían influido en su formación.

Como era de esperar, los entrevistados presentaron algunas sugerencias para mejorar el programa. En primer lugar, subrayaron la necesidad de contar con un mayor número de profesores de tiempo completo dedicado a la investigación y a la atención de estudiantes. En segundo lugar, insinuaron la posibilidad de íntegra el posgrado al Centro de Investigaciones de la Universidad Pedagógica (CIUP), "o de crear uno nuevo para desarrollar y publicar proyectos de investigación". En tercer lugar, insistieron en la urgencia de diseñar el aprendizaje a partir de proyectos de investigación con el apoyo de una formación teórica promovida en seminarios. $Y$ en cuarto lugar, solicitaron la ampliación de los recursos bibliotecarios de la Universidad y la participación de los estudiantes en las evaluaciones y en las decisiones del programa.

Los egresados fueron en general menos críticos que los estudiantes pero no por ello dejaron de apuntar observaciones importantes. Estuvieron de acuerdo en afirmar que el programa había respondido a sus expectativas y que a través de él habían alcanzado un mejor nivel de conocimientos y una mayor efectividad en el trabajo. Estimaron que sus profesores y la organización del posgrado eran "buenos", pero que últimamente se percibía una desorganización administrativa y un abandono en el desarrollo académico. Consideraron también que en la actualidad la mayoría de los estudiantes de posgrado no tiene una vocación intelectual sino un mero interés en ascender en el escalafón docente.

En cuanto a la calidad del programa, opinaron que si bien la información adquirida fue "excelente", no se logró un entrenamiento en investigación. Los cursos eran "magistrales" y toda la actividad se centraba en la elaboración de trabajos escritos y en la sustentación de contenidos y de lecturas en el salón de clase. En una perspectiva muy parecida a la de los estudiantes, los egresados juzgaron que los posgrados de la Universidad Pedagógica Nacional deberían reestructurarse con base en proyectos de investigación, donde los participantes pudieran identificar las dificultades relacionadas con la elaboración de los marcos de referencia, la comprobación de hipótesis y el manejo de datos. Pero también fueron conscientes de que las limitaciones de la investigación en el posgrado obedece a una situación mucho más amplia que rebasa los límites del programa. Recalcaron que el

\footnotetext{
${ }^{109}$ Los datos resumidos a continuación, están tomados de ibid., pp. 48-49, 54-59 y 63.
} 
problema de la investigación es institucional, esto es, de la Universidad como un todo: "realmente no se respira un ambiente científico entre los administradores, entre los profesores y menos aún entre los estudiantes".

Desgraciadamente, el trabajo de Murcia Florián no ofrece información sobre la carrera ocupacional de los egresados, rubro que no fue considerado en el cuestionario enviado a los estudiantes y a los que habían terminado sus estudios. Si bien se sabe que algunos estudiantes respondieron que el posgrado había contribuido al desarrollo de su profesión, y que los egresados estuvieron de acuerdo en afirmar que a través de él habían alcanzado una mayor efectividad en el trabajo, es poco lo que se conoce sobre la incidencia directa del programa en posibles ascensos o en cambios de ocupación y de ingresos. Tampoco se tiene un conocimiento preciso de si estos cambios han sido afines a las destrezas transmitidas por el programa. ¿Cuántos de ellos están dedicados a la investigación? ¿Cuántos a la enseñanza de materias relacionadas con la metodología y las técnicas de la investigación científica? ¿Cuántos trabajan en oficinas dedicadas a la formulación y al planeamiento de proyectos educativos? ¿Cuántos presentan una producción intelectual independiente del tutelaje personal del posgrado? A estas preguntas cuantitativas se podrían agregar otras de sabor más cualitativo. ¿Los egresados han logrado crear un clima propicio a la investigación en sus universidades? ¿Han cambiado los contenidos de las tradicionales cátedras de métodos y técnicas de investigación? ¿Han contribuido a la modernización de la recolección y análisis de datos en las oficinas de planeación y de formulación de programas educativos?

No es posible por ahora dar una respuesta exacta a estos interrogantes. Pero de todos modos existe información indirecta que de alguna manera ilustra la dirección general de las realizaciones ocupacionales de los egresados. El Cuadro No. 3, elaborado a partir de entrevistas informales, muestra la ubicación laboral de las 42 personas que han terminado sus estudios en Investigación Socioeducativa. Como era de esperar, un poco más de la tercera parte trabajan en universidades. Si a ellas se suman las cinco personas que en este momento se desempeñan como profesores de primaria y secundaria, se puede afirmar que el $50 \%$ de los egresados del programa tiene una vinculación directa con actividades docentes. Pero esta información es todavía muy gruesa y deja de lado aspectos cualitativos que sólo se logran con una pesquisa más detenida. Por ejemplo, varios profesores universitarios, cuyos estudios graduados habían sido patrocinados por sus respectivas instituciones, están actualmente al frente de las clases de metodología o de técnicas de investigación y han promovido la fundación o el mejoramiento de los centros de investigación de sus universidades.

Diez egresados trabajan en oficinas de planeación o en proyectos especiales relacionados con la formulación y seguimiento de programas de desarrollo social, entre los cuales los educativos ocupan un lugar importante. De los cinco extranjeros, procedentes de México, Panamá, Perú y Haití, cuatro han regresado a sus países y el otro adelanta en este momento su tesis de grado. Dos egresados más son investigadores de tiempo completo del Centro de Investigaciones de la Universidad Pedagógica (CIUP) en las áreas de historia y de sociología de la educación --en las cuales han publicado trabajos que empiezan a ser reconocidos por la comunidad intelectual ${ }^{110}$.

\footnotetext{
${ }^{110}$ Se carece, finalmente, de información sobre cinco personas que han terminado sus estudios, una de las cuales vive en el extranjero desde hace varios años.
} 
CUADRO No. 3

UBICACION OCUPACIONAL DE LOS EGRESADOS DEL POSGRADO DE INVESTIGACION SOCIO-EDUCATIVA

\begin{tabular}{lrr}
\multicolumn{1}{c}{ Instituciones } & No. & $\%$ \\
\hline Universidades & 15 & 36 \\
Educación primaria y secundaria & 5 & 12 \\
Oficinas de planeación (Planeación Nacional, & & \\
Ministerio de Educación, Distrito Especial, & & \\
Inravisión y Servicio Civil) & 6 & 14 \\
& & \\
Proyectos especiales (Ministerio de Hacienda & & \\
y asesorías en programas de desarrollo social & & \\
del Estado) & 4 & 9 \\
Extranjeros & 5 & 12 \\
Centros de investigación (ClUP) & 2 & 5 \\
Sin información & 5 & 12 \\
\hline \multicolumn{1}{c}{ Total } & 42 & 100
\end{tabular}

FUENTE: Entrevistas informales con los egresados adelantadas por el coordinador del posgrado de Investigación Socioeducativa. El nombre de los egresados con sus respectivas direcciones, se encuentra en J. Murcia Florián.

A juzgar por esta información, los objetivos del posgrado se han llevado a cabo en buena parte. La mayoría de sus egresados son profesores universitarios y algunos de ellos tienen a su cargo la docencia en métodos y técnicas de investigación y han promovido la institucionalización de la investigación en sus universidades. Otros, el $23 \%$, trabajan en oficinas donde se combina la investigación con la formulación y el seguimiento y administración de programas de desarrollo social. Estos rubros son, sin duda, una realización directa de dos de los fines que inicialmente se propuso el pos-grado. "Entrenar profesores de metodología" y desarrollar destrezas "en la elaboración y seguimiento de proyectos de planeación educativa". Pero en materia de investigación como actividad exclusiva y permanente, los resultados son pobres: sólo dos egresados se desempeñan como investigadores de tiempo completo. Sin embargo, esta limitación' tiene que ver más con la escasez de centros de investigación dedicados a esta tarea que a ausencias mismas del posgrado. Estrictamente hablando, el CIUP es el único centro especializado en investigación educativa en el país, los demás centros, públicos o privados, ofrecen una variedad temática bastante amplia donde la educación es apenas un interés entre muchos otros (salud, vivienda, ingresos, servicios públicos, etc.).

Las entrevistas realizadas por el autor de la evaluación con funcionarios del Instituto Colombiano para el Fomento de la Educación Superior (ICFES) y con profesores de la Universidad de Antioquia, donde existe un programa semejante, plantearon la importancia de sostener el programa de Investigación Socioeducativa. Para unos y otros, los recientes cambios en el escenario de la educación y la introducción de tecnologías de otros países en las instituciones colombianas, han creado la necesidad de contar con personal calificado en materia de investigación educativa con especial sensibilidad en las variables 
socio-culturales. Indicaron que la implantación de estas tecnologías en los países en desarrollo estaba resintiendo aún más la endeble estructura de sus sistemas educativos. Si bien no definieron el contenido y dirección de estos problemas, en los entrevistados era evidente el temor por la dependencia científico-técnica cuando no se dispone de los instrumentos para impulsar una política propia de investigación. Así, con un fuerte acento nacional afirmaron:

“...La transferencia de tecnología, además de ignorar los valores culturales de nuestras clases sociales, obstruye la capacidad de los pueblos para investigar, crear y desarrollar procesos teórico-prácticos de la realidad"111.

La capacidad de manejo de las situaciones propias, la competencia crítica y la habilidad para discernir las experiencias extranjeras, demandan -a su juicio la existencia de un programa dedicado a la formación de investigadores en educación. Pero todo ello a condición de que se lo haga a partir de una familiaridad con las complejidades de la ciencia, requisito sin el cual no puede adelantarse un conocimiento cabal de los problemas nacionales ni mucho menos intentar su transformación.

\section{Bases para una reforma del posgrado}

De las anteriores experiencias del posgrado de Investigación Socioeducativa, se desprenden varias lecciones para el futuro. La primera de ellas, relacionada con la organización docente, se puede resumir en pocas palabras: no debe sostenerse una estructura académica alimentada en su mayor parte por profesores que apenas tienen obligaciones con sus estudiantes. Debe ampliarse la nómina de profesores de tiempo completo para atender las demandas del cuerpo estudiantil. La vinculación de docentes por unas pocas horas a la semana, no obstante su capacidad y sus logros individuales, no alcanza a cubrir las múltiples exigencias vinculadas a la formación de investigadores dirigidos a enriquecer la teoría o a diseñar, evaluar y administrar programas de acción social.

La segunda lección se refiere a la estructura curricular. Hay un acuerdo general de que el programa debe organizarse alrededor de actividades de investigación y no de materias distribuidas en semestres ${ }^{112}$. Si el objetivo es la formación de investigadores, toda la dinámica del posgrado debe estar orientada hacia las prácticas de investigación donde profesores y estudiantes esclarezcan las dificultades del oficio. Se hace necesario afrontar en forma directa los problemas vinculados al archivo, al trabajo de campo, a la manipulación de estadísticas y al diseño y análisis de encuestas. Los seminarios deberán familiarizar a los estudiantes con las grandes tradiciones de pensamiento y rescatar el papel activo de la teoría en la orientación de las investigaciones en marcha.

Pero todo esto debe ir acompañado de una nueva modalidad en el reclutamiento de los aspirantes. Aunque ahora se propone una formación más "desescolarizada", también se exige una mayor concentración en las labores de biblioteca y en las tareas relacionadas con el trabajo de campo (en el sentido amplio de prácticas de investigación). Por lo tanto - --y esta es la tercera lección-, los alumnos deben contar, además de la vocación intelectual, con las facilidades necesarias para invertir todo su tiempo en su

\footnotetext{
${ }^{111}$ J. Murcia Florián, op. cit., p. 63.

${ }^{112}$ Este es también el punto de partida de la propuesta de una maestría en investigación educativa elaborada por tres investigadores del CIUP. Ver Alberto Martínez Boom. Leonor Zubieta y César A. Vera, Propuesta para un magíster en investigación educativa (Bogotá: Universidad Pedagógica Nacional - Centro de Investigaciones (CIUP), 1982). Los dos primeros autores son egresados del posgrado de Investigación Socioeducativa.
} 
formación teórica y práctica. Con esta estrategia curricular, las personas que están comprometidas en jornadas laborales extra-universitarias, difícilmente podrían lograr un aprovechamiento escolar y a la primera oportunidad optarían por abandonar el programa.

La cuarta y última lección tiene que ver con la Universidad como institución de educación superior. La Universidad Pedagógica Nacional debe emprender una política agresiva de actualización de su biblioteca y de su centro de documentación. Debe expandir rápidamente su colección de revistas nacionales y extranjeras y su fondo de problemas colombianos y latinoamericanos. Cabe recordar una vez más, que el nivel académico de una institución no depende solamente de las características de sus profesores, sino también de la calidad de sus recursos educativos. Los egresados llevarán al mercado de trabajo la huella de sus instituciones, esto es, las destrezas de sus profesores y el monto y calidad de los recursos que encontraron en sus universidades y acompañaron su formación profesional.

Como consecuencia de lo anterior, algunos objetivos del posgrado deben ser redefinidos. Si en un principio se subrayó la necesidad de formar profesores de metodología para las facultades de Educación, hoy el énfasis debe estar puesto en la formación de investigadores propiamente dichos. Las transformaciones que es necesario emprender en este momento en aquellas instituciones, están relacionadas con la promoción de investigaciones concretas y no con la difusión meramente formal de sus pasos y de sus bondades. Además de enseñar métodos y técnicas de investigación, hay que demostrar que ella es posible y que sus resultados ofrecen contribuciones significativas para la institución, para la ciencia y para la práctica social.

Pero si el posgrado debe emprender estas reformas, también debe rescatar sus mejores logros. No hay que olvidar sus constantes esfuerzos por integrar la teoría al proceso de investigación, su enseñanza de los clásicos de la ciencia social y su permanente discusión de las complejidades de la estructura y de la dinámica sociocultural. Un programa dirigido a capacitar investigadores no puede eludir la formación teórica de sus estudiantes, pues es sabido que nadie consigue investigar a menos que posea conceptos, enfoques y perspectivas generales que le permitan organizar el caos de la "realidad". El posgrado debe por lo tanto plantearse como uno de sus ideales impulsar investigaciones teóricamente relevantes además de social-mente significativas. A pesar de que este ideal es difícil de alcanzar, su presencia inducirá a estudiantes y profesores a estar más atentos a los necesarios equilibrios exigidos por cada situación. De todos modos, quien no tenga conciencia de las permanentes tensiones entre estas dos demandas, siempre estará en peligro de caer en un teoricismo vacuo o en un pragmatismo estrecho.

\section{Nueva estrategia curricular}

Las conclusiones anteriores reclaman una nueva estrategia curricular. La experiencia descrita muestra claramente que es necesario organizar las actividades del posgrado a partir de tareas concretas de investigación y de un manejo funcional de la teoría y de los marcos de referencia adecuados al estudio de los procesos educativos. Debe dejarse de lado una formación centrada exclusivamente en el salón de clase y abordar desde un comienzo la variada riqueza del trabajo de campo, que nunca logra ser remplazada por lecturas o por las abundantes formalizaciones de los manuales de metodología. 
La primera exigencia para llenar este cometido, es la creación de un clima intelectual en el seno del posgrado. Esta tarea no es fácil, sobre todo cuando se recuerda que la Universidad Pedagógica Nacional no tiene tradición en materia de investigación. Su organización académica —-tomo la de la mayoría de las universidades del país—, gira alrededor de labores meramente docentes y administrativas. Allí la producción intelectual y la investigación son escasas y dispersas y nunca logran traducirse en acciones sistemáticas y acumulativas. El profesor se define más como un funcionario que debe cumplir con ciertas demandas laborales estipuladas por la institución que como un hombre de conocimientos cuya función es la transmisión de un saber renovado y en permanente evolución. Esta situación que tiende a autorreproducirse, sólo podrá ser afrontada con un grupo docente altamente calificado y con experiencia en investigación. En esta fase inicial, el programa deberá contar con un mínimo de tres profesores de tiempo completo, cada uno de los cuales tendrá a su cargo uno o dos proyectos de investigación que adelantará con la participación de estudiantes ${ }^{113}$. La producción de estos docentes y las discusiones que ellas susciten, comenzarán a crear un clima positivamente orientado hacia la búsqueda de nuevos conocimientos. Pero lo que es todavía más importante, y que tiende a olvidarse por hacer parte del sentido común, es que si los profesores no investigan, difícilmente lo harán sus alumnos, o, lo que es lo mismo, si el profesorado de un programa de graduados no ofrece producción intelectual alguna, carece de legitimidad para exigírsela a sus estudiantes.

Pero el reducido número de profesores de tiempo completo difícilmente podrán satisfacer las múltiples demandas asociadas a la formación de investigadores. Ellos apenas lograrán absorber unos pocos estudiantes, no más de tres por proyecto, que estén interesados en los aspectos cualitativos de la investigación educativa. Las limitaciones mismas de la Universidad en recursos técnicos para adelantar estudios cuantitativos, lleva forzosamente a que sus docentes subrayen la investigación histórica, la etnográfica o la de carácter teórico. Sin embargo, este vacío puede ser llenado por otras instituciones que la Universidad Pedagógica Nacional debe comenzar a ver como los sitios de práctica de sus estudiantes graduados: los centros de investigación públicos y privados. De la misma forma que las facultades de medicina tienen los hospitales, las de sicología las clínicas siquiátricas, las de derecho los juzgados y los consultorios jurídicos como sus lugares naturales de entrenamiento profesional, los programas de graduados en investigación deberán acudir a los centros de investigación en pos de las destrezas requeridas por su trabajo. A ellos podrán asistir los alumnos interesados en la investigación social empírica, donde el manejo de un volumen amplio de datos requiere de un entrenamiento diferente al de la investigación cualitativa.

En los centros de investigación, especialmente en los mejor dotados y en los de mayor tradición, los estudiantes no sólo tendrán acceso al equipo técnico necesario para el procesamiento de datos, los computadores, sino también -y esto es tal vez lo más importante-, tendrán la oportunidad de participar en las diversas etapas de una investigación de gran tamaño. Podrán observar las negociaciones del centro con sus clientes, la definición del problema de investigación, su adecuación al presupuesto, la traducción de los objetivos a un lenguaje de variables que permita enunciar los datos requeridos, el diseño de los instrumentos de recolección de datos, la recolección de los

\footnotetext{
${ }^{113}$ Esta demanda está consignada, por lo demás, en la reglamentación de estudios de posgrado de la Universidad Pedagógica Nacional, donde se estipula claramente que "cada programa de posgrado deberá tener adscritos un mínimo de tres profesores de tiempo completo, uno de los cuales será el encargado de la coordinación”. Ver artículo octavo del Acuerdo 44 del Consejo Directivo de mayo 10 de 1979 .
} 
mismos en el terreno, su clasificación y análisis, y la organización y presentación del informe final.

Estas etapas, muchas de las cuales se desarrollan simultáneamente, harán comprender al estudiante que la investigación social moderna, especialmente aquella asociada al análisis de encuestas, es una actividad organizada, en la cual un grupo de personas bajo la dirección de un jefe de proyecto desempeña diferentes papeles encaminados a alcanzar un producto final. Cada persona hacer su labor para llenar las necesidades del conjunto y asegurar el éxito de la investigación. Al tomar conciencia de estas actividades, el investigador novel no solamente se familiarizará con las destrezas requeridas en cada fase, sino también con las habilidades necesarias para organizar y orientar un equipo de investigación. Pues sólo quien posea experiencia en las diversas operaciones de una investigación puede efectivamente dirigir un proyecto y coordinar el trabajo de los demás ${ }^{114}$.

Este contacto directo con un centro de investigación contribuirá también a la socialización laboral del joven investigador. Allí establecerá relaciones con directores de otros centros, con investigadores experimentados y con organismos nacionales o internacionales que podrán orientarlo en su futuro desempeño ocupacional. Encontrará, así mismo, la posibilidad de adelantar su tesis de grado, bien sea a través de la información ofrecida por las investigaciones en las cuales participó, o a través de los datos acumulados por el centro a lo largo de los años.

Pero las relaciones Universidad-centros de investigación tienen una faceta mucho más enriquecedora que hasta ahora apenas ha sido aprovechada. Mientras que los centros se han dedicado a la investigación aplicada y a las labores de consultoría, las universidades han tendido a recalcar la investigación básica y las discusiones teóricas. Estos énfasis han llevado a ambas instituciones a descuidar, si no a desconocer totalmente, el trabajo realizado por cada una de ellas. Los centros desechan la producción universitaria por "retórica" y las universidades rechazan la labor de aquellos por "empírica", limitada y ateórica. Unos y otros llevan, por supuesto, su verdad. Tradicionalmente los centros privados han reunido sus datos para llenar las necesidades de sus clientes —el Estado, las empresas y los organismos internacionales-, sin atender al significado más amplio de sus resultados; y las universidades se han debatido en una oscura prosa donde apenas se definen los conceptos y las temáticas objeto de estudio. Estos estilos opuestos de trabajo intelectual deben terminar, y la mejor forma de rescatar sus cualidades es creando las condiciones para establecer un diálogo fructífero entre las instituciones que poseen los recursos y las facilidades técnicas para la manipulación de datos y las que tienen una preocupación más amplia y general por la relevancia de los hallazgos. Las universidades pueden convertirse en un excelente grupo de referencia crítico para la reunión y uso indiscriminado de información, y a su vez ellas pueden aprender de sus colegas de los centros que el conocimiento adquirido con rigor metodológico facilita la construcción de teorías más concretas y operativas ${ }^{115}$. Los centros, por su lado, comenzarán a despojarse de su tinte utilitarista asociado a una economía de mercado, y abrirán las puertas para

\footnotetext{
${ }^{114}$ Estos párrafos deben mucho al famoso memorando de Paul F. Lazarsfeld y Robert K. Merton, “A Professional School for Training in Social Research", escrito en 1950 y publicado por primera vez en 1972. Ver Paul F. Lazarsfeld, Quaiitative Analysis: Historical and Critical Essays (Boston: Allyn and Bacon, 1972), pp. 361-391. Una exposición más amplia del contexto organizativo de la investigación social empírica, se encuentra en Herbert Hyman, Diseño y análisis de las encuestas sociales (Buenos Aires: Amorrortu, 1971), pp. 58-71.

${ }^{115}$ Este trabajo conjunto podría iniciarse haciendo "análisis secundarios", esto es. retomando con fines académicos los datos de una o varias investigaciones ya realizadas. Como es sabido, una mente teóricamente sensible puede extraer información valiosa de las investigaciones aplicadas y servirse de ella para sugerir hipótesis o poner en cuestión antiguos marcos de referencia. 
desarrollar intereses académicos que contribuirán a formar los recursos humanos que ellos mismos van a requerir en el futuro próximo.

En una perspectiva más general, las relaciones universidad-centros de investigación no sería más que una estrategia dirigida a racionalizar el uso de los escasos recursos científico-técnicos de un país subdesarrollado. Los investigadores experimentados tomarían a su cargo unos estudiantes graduados para entrenarnos en las destrezas requeridas por el oficio, y éstos a su vez serían de gran ayuda para cubrir las tareas cotidianas de los centros: recolección de datos, clasificación, tabulación, organización de tablas estadísticas, etc. Sería entonces una empresa donde centros, universidades y estudiantes colaborarían en el desarrollo de indagaciones de valor práctico inmediato (investigaciones aplicadas) y de trabajos destinados a realizar aportes a un cuerpo organizado de conocimientos (investigaciones básicas).

Pero todo esto no significa que el posgrado en Investigación Socioeducativa abandonará el exclusivismo del salón de clase por el de las prácticas de investigación. Aquí, como en toda política académica relacionada con las ciencias sociales, es necesario guardar un sano equilibrio entre la adquisición de las destrezas y la comprensión de su significado. Sí la investigación no puede llevarse a cabo sin conocer previamente sus etapas, el conocimiento de éstas no remplaza el dominio de las orientaciones teóricas que confieren sentido a los datos que se han logrado reunir. El joven investigador debe saber manejar conceptos y debe conocer la forma de relacionarlos y de traducirlos a problemas de investigación, pues —como los expertos siempre lo recuerdan-, uno de los momentos más difíciles de la investigación social es la elaboración de un marco de referencia funcional al problema que se desea estudiar.

Para responder a estas necesidades de formación teórica, el posgrado diseñará un conjunto de seminarios para debatir la perspectiva y el núcleo conceptual de diversas ciencias interesadas en la investigación socio-educativa. A lo largo de los dos años, se ofrecerán seminarios sobre sociología, economía e historia de la educación a cargo de los profesores de planta del programa. En ellos se discutirán los trabajos más significativos de cada especialidad y se buscará familiarizar al estudiante con las controversias teóricas y metodológicas que informan su actual desarrollo. La obligatoriedad de estos seminarios está asociada a los intereses temáticos de los estudiantes y su objetivo estará dirigido a contribuir a solucionar los problemas que surjan en su trabajo de investigación. Esta es la razón por la cual los profesores de tiempo completo del posgrado tendrán que ser personas con experiencia en el manejo de teorías y con pericia en los procedimientos de indagación social.

Estos seminarios adquieren una mayor significación en el contexto de las universidades pedagógicas, donde ha tomado fuerza la idea de que el trabajo en grupo soluciona los problemas de interpretación y análisis. Con frecuencia se piensa allí que la suma de las perspectivas del sicólogo, del sociólogo, del economista y del historiador ofrecerá una información más rica sobre los fenómenos educativos. Pero la realidad es bien distinta. En educación como en cualquier otro campo de la vida social, sólo puede hacer investigación de calidad quien parte de una ciencia, y esto — como lo señala el sentido común-, está estrechamente asociado con el dominio que se tenga de ella.

Y en caso de que se desee alcanzar una síntesis a partir de los hallazgos de diversas especialidades, es necesario tener conciencia de lo que se está mezclando y poseer una estrategia integradora que permita organizar los resultados. La base de esta estrategia la proporciona generalmente una de las ciencias en cuestión, como lo muestran los 
repetidos esfuerzos de la sociología, la antropología y la historia por lograr una comprensión lo más compacta posible del mundo social. Contra lo que comúnmente se piensa entonces, la búsqueda de la totalidad no ahorra en ningún momento la familiaridad con las ciencias particulares, sino que por el contrario, demanda un conocimiento más profundo de ellas y mucho más puntual del que usualmente presenta el hombre informado. Es por ello que los usuales encuentros de especialistas en educación y la suma aritmética de sus contribuciones en un volumen, no van más allá de lo que el sociólogo Karl Mannheim recordaba como la "síntesis del encuadernador". Aquí el operario encargado de coser el lomo del libro resulta uniendo mecánicamente lo que sus autores debieron ensamblar orgánicamente ${ }^{116}$.

La recuperación del reequilibrio entre la teoría y la investigación en el posgrado, lleva también a una redefinición del papel de los clásicos de la ciencia social en la formación de investigadores en educación. Como se sabe, los clásicos proporcionan un modelo para el trabajo intelectual y ofrecen un andamiaje conceptual para obtener una explicación más coherente de los fenómenos sociales. Previenen contra el parroquialismo intelectual y promueven las discusiones de importancia para el avance del conocimiento. Quien se ha formado a la sombra de espíritus penetrantes como Emile Durkheim, Max Weber, Georg Simmel, Thorstein Veblen o Karl Mannheim, lleva una marca indeleble que usualmente se expresa en el buen sentido y en el juicio certero ${ }^{117}$. - Con diferencias de grado, en estos autores está siempre presente la intención de lo general sin olvidar la singularidad de los fenómenos, o dicho de otra manera, tienen la rara cualidad de extraer conclusiones generales después de adelantar agudos escrutinios de lo particular. La discusión del legado de los clásicos será por lo tanto un instrumento creativo para ampliar el contexto y la perspectiva de la investigación social y no un mero adorno retórico para salpicar de erudición los informes de investigación.

La estrategia curricular aquí diseñada vuelve a restablecer el necesario balance entre la práctica y la reflexión conceptual. Evita el énfasis retórico de los anteriores programas y rescata la tradición teórica asociada a los mejores departamentos de graduados. Sugiere el uso de la capacidad científico-técnica de que dispone el país y demanda un empleo más racional de los recursos inter-institucionales. Pide, finalmente, que haya una redefinición de la política universitaria en materia de reclutamiento docente, con el objeto de crear un clima favorable al desarrollo intelectual, a la investigación y a la búsqueda de nuevos conocimientos. Pero al final, como ocurre con toda idea que busca traducirse en acción, el éxito de la presente estrategia dependerá del compromiso real que la institución y sus integrantes tengan con los procesos que se intentaron describir en las páginas anteriores.

SUMARIO: El artículo busca evaluar el camino seguido por el programa de Investigación socioeducativa, el único Posgrado en Ciencias Sociales de la Universidad Pedagógica Nacional. Describe los sucesivos cambios curriculares, las características de profesores y estudiantes para pasar a discutir sobre la inserción ocupacional de los egresados de estos estudios. Pretende aportar elementos de análisis tendientes a promover evalua-

\footnotetext{
${ }^{116}$ Ver el "Prólogo" de K. Mannheim al libro de Viola Klein, EL carácter femenino (Buenos Aires, Paidós, 1965), p. 18. Esta obra es un excelente ejemplo de la integración de diversas perspectivas científicas — biológicas, sicológicas, históricas, antropológicas y sociológicas - para ilustrar las facetas de un problema claramente delimitado: el carácter femenino. La autora parte de una estrategia histórico-sociológica que le permite organizar las distintas maneras de ver "lo femenino" en la sociedad contemporánea.

${ }^{117}$ Hans Zetterberg, Teoría y verificación en sociología (Buenos Aires: Nueva Visión, 1968), p. 16.
} 
ciones de otros programas de posgrados, en cuanto estos niveles conforman una parte del sistema educativo, en franca expansión en el país.

DESCRIPTORES: Educación, Estudios Sociales, Evaluación educacional, Evaluación del Programa, Colombia. 